\title{
Fecal Microbiota Transplantation as an Effective Treatment for Carbapenem-Resistant Klebsiella pneumoniae Infection in a Renal Transplant Patient
}

\author{
Junpeng Wang ${ }^{1,2, *}$ \\ Xin $\mathrm{Li}$ (D) $^{3,4, *}$ \\ Xiaoqiang $\mathrm{Wu}^{\prime}$ \\ Zhiwei Wang' \\ Xuan $\mathrm{Wu}^{\prime}$ \\ Shanmei Wang ${ }^{5}$ \\ Gaopeng Jing' \\ Tianzhong Yan'
}

'Department of Urology, Henan Provincial People's Hospital, Zhengzhou University People's Hospital, Henan

University People's Hospital, Zhengzhou, People's Republic of China; ${ }^{2}$ Department of Organ Transplantation, Zhujiang Hospital, Southern Medical University, Guangzhou, People's Republic of China; ${ }^{3}$ Department of Pathophysiology, School of Basic Medical Sciences, Zhengzhou University, Zhengzhou, People's Republic of China; ${ }^{4}$ Provincial Cooperative Innovation Center for Cancer Chemoprevention, Zhengzhou, People's Republic of China; ${ }^{5}$ Department of Clinical Microbiology, Henan Provincial People's Hospital, People's Hospital of Zhengzhou University, Zhengzhou, People's Republic of China

*These authors contributed equally to this work
Correspondence: Tianzhong Yan Department of Urology, Henan Provincial People's Hospital, Zhengzhou University People's Hospital, Zhengzhou, Henan Province, 450003, People's Republic of China

Email ytz460@hotmail.com
Background: In renal transplant recipients, carbapenem-resistant Klebsiella pneumoniae (CRKP) infection is a common complication, and usually associated with severe clinical outcomes due to a lack of effective treatment.

Case Presentation: A 37-year-old woman with CRKP infection one month after kidney transplantation was involved in this study. Microbial characteristics of fecal samples from the patient were analyzed. Fecal microbiota transplantation (FMT) was performed for treating the CRKP infection. One week after FMT, the patient's urine and anal swab cultures returned negative for CRKP, and 17 days after FMT, the incision showed complete healing. Moreover, the patient had no symptoms of infection two months after FMT. Alpha diversity analyses showed that before FMT, the patient was associated with obviously lower species richness and diversity than the donor, which significantly increased at one week, three weeks and two months after FMT. Beta diversity analyses showed that though the patient's microbial community post-FMT still differed from the donor composition, their distances decreased visibly, especially at one week and three weeks after FMT. Obvious shift in microbial composition could be observed before and after FMT. The microbial composition of the patient post FMT resembled the donor composition. Relative abundance of genera such as Phascolarctobacterium and Lachnoclostridium increased after FMT, while the relative abundance of Klebsiella significantly decreased.

Conclusion: This study demonstrated the therapeutic effect of FMT on infections caused by CRKP for a renal transplant patient. Further studies are required to confirm the findings of this study.

Keywords: carbapenem-resistant Klebsiella pneumoniae, infection, fecal microbiota transplantation, gut microbiota

\section{Background}

Infections caused by carbapenem-resistant Klebsiella pneumoniae (CRKP) have been a serious health problem worldwide ${ }^{1}$ and are usually associated with poor therapeutic outcomes due to the fact that effective strategies are limited. ${ }^{2}$ In organ transplant recipients treated with immunosuppressed agents, CRKP infections can be lifethreatening.

Here, we report the use of fecal microbiota transplantation (FMT) in a renal transplant recipient with CRKP infection. The FMT achieved positive clinical outcomes, and resulted in increased richness and diversity of gut microbiota and reduced relative abundance of Klebsiella. 


\section{Case Presentation}

A 37-year-old Chinese woman suffering from end-stage renal disease underwent a cadaveric kidney transplantation in November 2018. Postoperative recovery was uneventful and the serum creatinine level of the patient returned to normal three days after the transplantation. On postoperative day 8 , the patient experienced acute rejection, which was treated with high-dose prednisone and plasma exchange, and after treatment, her renal function became normal. The patient developed CRKP infection one month after the kidney transplantation. The CRKP could be detected in incision secretion, urine, blood and anal swab cultures of the patient, and resulted in impaired surgical incision healing. Multi-locus sequence typing (MLST) of seven sets of DNA fragments derived from housekeeping genes ${ }^{3}$ was performed to examine the genetic diversity of CRKP strains isolated from the incision, urine, and fecal samples of the patient, and the results revealed that all the three strains were exclusively grouped into ST11 (Table S1 and Figure S1). The hypermucoviscous and hypervirulent nature of the Klebsiella pneumoniae were also checked using the "string test" 4 and qRTPCR, respectively. The results showed that the CRKP isolate exhibited a positive string test (Figure S2), and expressed virulence-associated genes (rmpA2, ybtS, iucA, rmpA and peg344; Table S2 and Figure S3), indicating its hypermucoviscous and hypervirulent phenotype. Since the CRKP isolated from the patient was resistant against most antibiotics except tigecycline, fosfomycin and polymyxin (Table S3), the combination treatment of with tigecycline $(50 \mathrm{mg} / 12$ hours), fosfomycin (4 g/8 hours) and meropenem (1 2 g/8 hours) and was utilized for treating the infection. The detailed treatment timeline for the CRKP infection is shown in Figure 1. After 46 days of antibiotic treatment, the incision of the patient could still not heal. The CRKP converted to be negative in the patient blood cultures, but positive in the purulent incision secretion, urine and anal swab cultures. The patient had normal body temperature and white blood cell (WBC) count $\left(4.6 \times 10^{9} / \mathrm{L}\right)$, but the $\mathrm{C}$-reactive protein (CRP) level was elevated $(24.79 \mathrm{mg} / \mathrm{L})$. Symptoms of urinary tract infection such as frequent and urgent urination, and pelvic pain could also be observed. Under this condition, FMT was performed, and antibiotics were stopped one day before FMT. Preoperatively, the patient took proton pump inhibitor, and polyethylene glycol electrolyte solution was given for bowel preparation. Washed microbiota suspensions from a healthy volunteer (screening protocol was shown in Table S4) obtained from fmtBank.org were used for FMT. The preparation of the washed microbiota suspensions was conducted as described previously. ${ }^{5}$ Briefly, $10 \mathrm{~cm}^{3}$ (about $1.0 \times 10^{13}$ bacteria) of the final precipitated microbiota is the basic unit dose for clinical use, and the volume ratio of the final precipitation/vector solution for making suspensions is $1: 2$. In this study, 10.3 basic units of the washed microbiota suspensions (about $1.03 \times 10^{14}$ bacteria) were given to the patient via nasogastric tube. ${ }^{5}$ The patient was not given any antimicrobial agent postoperatively. One week after FMT, the patient's urine and anal swab cultures returned negative for CRKP. The patient's incision began to heal three days after FMT, and showed complete healing 17 days after FMT (Figure 2A). The patient tolerated the FMT procedure without complications including abdominal pain, diarrhea and fever. Moreover, there was no obvious increase in serum creatinine and blood urea nitrogen (BUN) after FMT (Figure 2B), suggesting FMT did not have adverse effect on the patient's renal function. The patient had no symptoms of infection two months after FMT, indicating a positive clinical outcome.

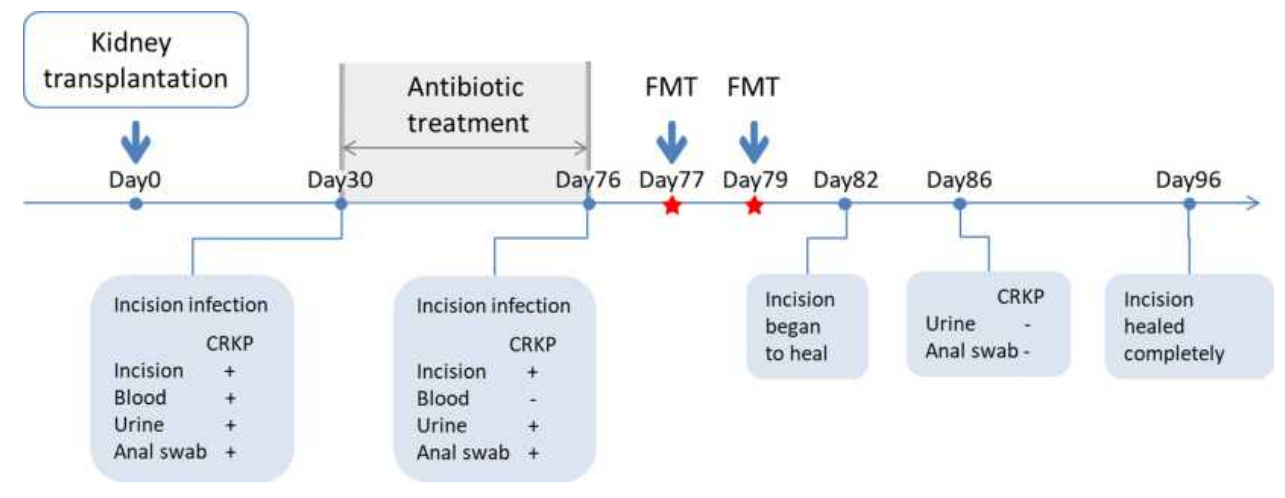

Figure I Treatment timeline for CRKP infection. 
A

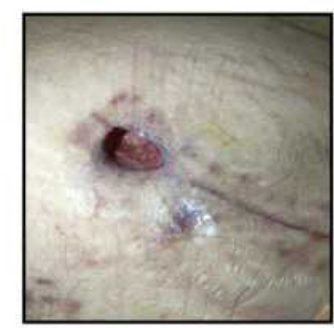

14d before FMT

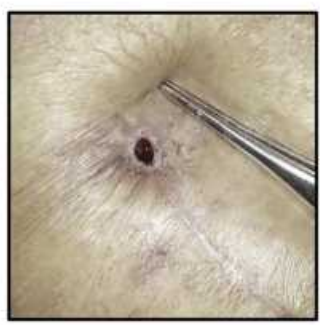

3d post FMT

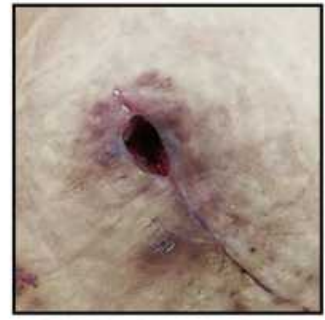

7d before FMT

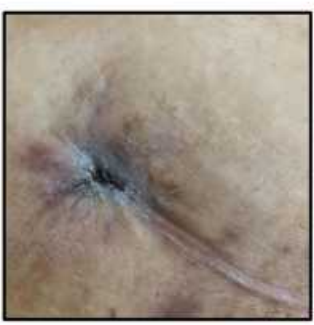

4d post FMT

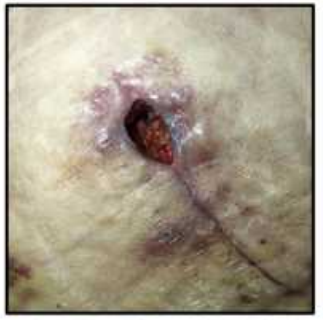

1d before FMT

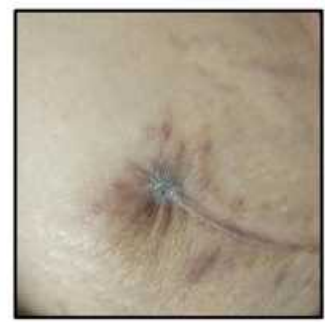

17d post FMT
B
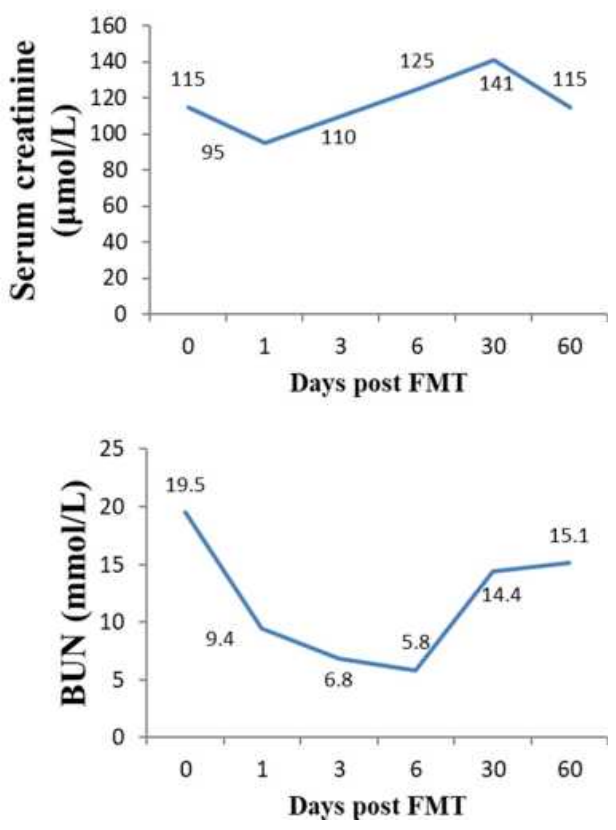

Figure 2 Surgical incision and renal function indexes in the patient post-FMT. (A) Surgical incision for kidney transplantation in the patient. (B) Serum creatinine and blood urea nitrogen (BUN) levels.

We performed a microbiota analysis of the fecal samples from the donor and the patient. Alpha diversity analyses showed that before FMT, the patient was associated with obviously lower species richness (Chaol index, Figure 3A) and diversity (Shannon index, Figure 3B) than the donor, which significantly increased at one week, three weeks and two months after FMT (Figure $3 \mathrm{~A}$ and $\mathrm{B}$ ), indicating that FMT could lead to an increase in the richness and diversity of the patient fecal microbiota. Next, beta diversity analysis was calculated using weighted and unweighted UniFrac distances, and visualized via principle coordinate analyses (PCoA) to compare microbial communities between samples. Both the weighted (Figure 3C) and unweighted (Figure 3D) UniFrac methods revealed significant divergence of the microbial communities between the donor and the patient before FMT. It should be noted that though the patient microbial community post-FMT still differed from the donor one, their distances visibly decreased, especially at one week and three weeks after FMT (Figure 3C and D).

Furthermore, we analyzed the microbial compositions in detail, and the results were visualized as cumulative histograms (Figure 4A). At genus level, Prevotella_9 (relative abundance 35.9\%), Ruminococcaceae_NK4A 214_group (relative abundance 12.9\%) and Faecalibacterium (relative abundance 10.0\%) accounted for the major bulk of the donor microbiota, while Bacteroides (relative abundance 60.6\%) and Klebsiella (relative abundance $38.9 \%$ ) were predominant in the recipient's sample, which accounted for nearly $90 \%$ of the total taxa. At one and three weeks after FMT, Phascolarctobacterium increased significantly in relative abundance (28.8\% and 26.1\%), and became the most dominant taxon. Besides Phascolarctobacterium, the proportions of [Ruminococcus]_gnavus_group, Alistipes, and Lachnoclostridium were also observed to increase after FMT. Meanwhile, at two months after FMT, Bacteroides, Lachnoclostridium and Phascolarctobacterium were the predominant populations in the fecal samples of the patient. Results of the cluster analysis showed that the fecal microbial composition of the patient following FMT was more similar to the donor microbial composition than that of the patient collected prior to FMT (Figure 4A). It should be noted that the relative abundance of Klebsiella significantly decreased at one week after FMT $(61.15 \%$ vs $1.5 \%)$, and remained low at three weeks (1.4\%) and two months $(6.0 \%)$ after FMT (Figure 4B). Moreover, the relative abundances of Ruminococcus, Coprococcus, and Clostridium were negatively correlated with that of Klebsiella, indicating that these genera may competitively interact with Klebsiella (Figure 4C). 
A

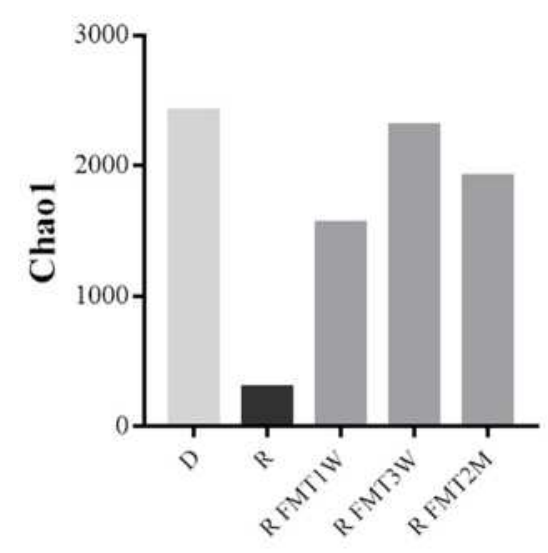

C

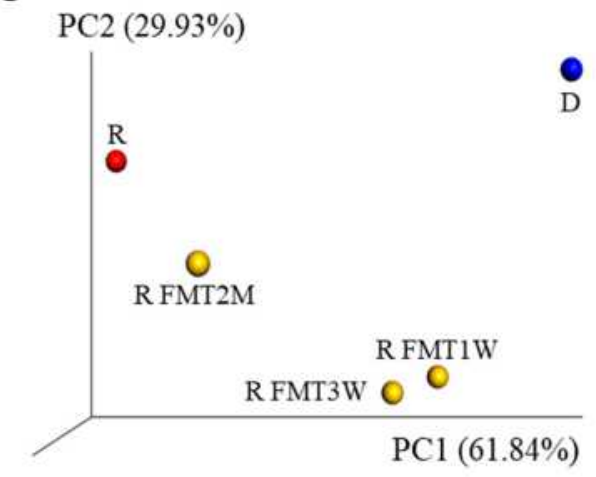

PC3 $(7.42 \%)$
B

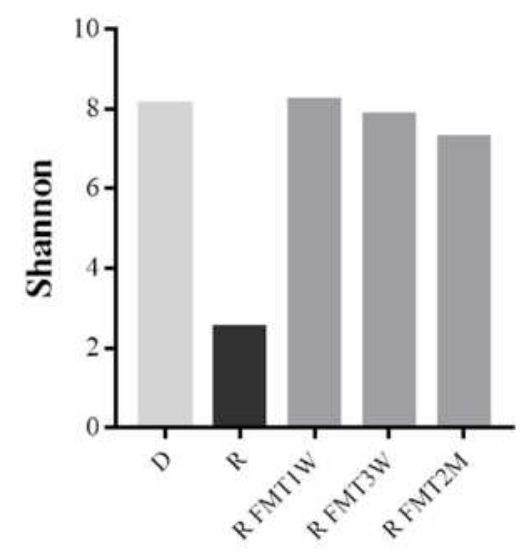

D

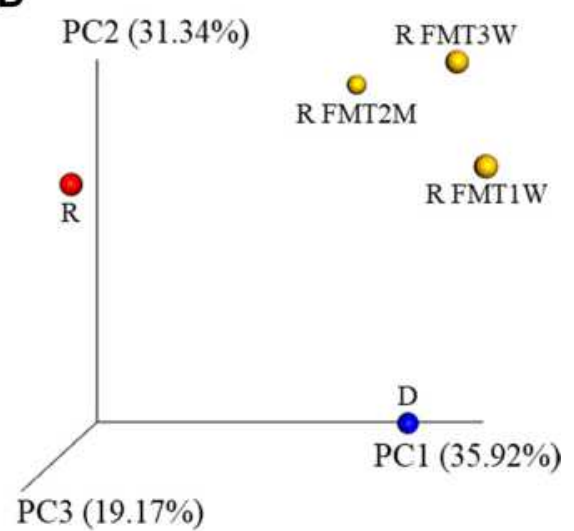

Figure 3 Alpha and beta diversity of the gut microbiota of the donor and the patient pre- and post-FMT. Histograms compared Chaol index (A) and Shannon index (B) of the fecal microbiome in the donor and the patient pre- and post-FMT. PCoA plots showed the microbial community variation of the donor and the patient pre- and postFMT based on weighted UniFrac distance (C) and unweighted UniFrac distance (D). FMT IW, FMT 3W and FMT 2M represented I week, 3 weeks and 2 months after FMT. Abbreviations: $\mathrm{D}$, donor; $\mathrm{R}$, recipient.

\section{Discussion and Conclusions}

In this study, we tried to use FMT to treat the CRKP infection in a renal transplant patient. Because CDI is underdiagnosed and undertreated in China, a non-profit organization named Chinese fmtBank (fmtbank.org) provides rescue FMT service for patients with recurrent or refractory CDI across the whole nation. ${ }^{6}$ Besides, the indications of FMT from fmtBank in practice also include antibiotic-associated diarrhea (AAD) and other refractory intestinal infections. Under this background, we performed FMT to treat the CRKP infection with the support of fmtbank.org. Given that the renal transplant patient in this study requires long-term immunosuppression, had a possibility of increased permeability of intestinal mucosa, and was more susceptible to the potential infection from FMT, we performed washed microbiota transplantation, which has been demonstrated to be safer, more precise and more quality-controllable than the crude FMT by manual..$^{7-10}$ FMT was first reported to be utilized for the treatment of described severe diarrhea in 4th century China, and has now been used worldwide to successfully treat patients with recurrent Clostridioides difficile infection (CDI), which is unresponsive to antibiotic treatment. ${ }^{1}$ Moreover, the use of FMT in critically ill patients with antibiotic-associated diarrhea also obtained good clinical outcomes without infectious complications. ${ }^{6}$ Currently, FMT has also been attempted for treating the urinary tract infection (UTI) caused by extended-spectrum beta-lactamase-producing $(\mathrm{ESBL}+)$ Klebsiella pneumoniae in a renal transplant recipient. After being treated with FMT, the UTI in the patient was well controlled, and the ESBL+ Klebsiella pneumoniae was eradicated, 
A

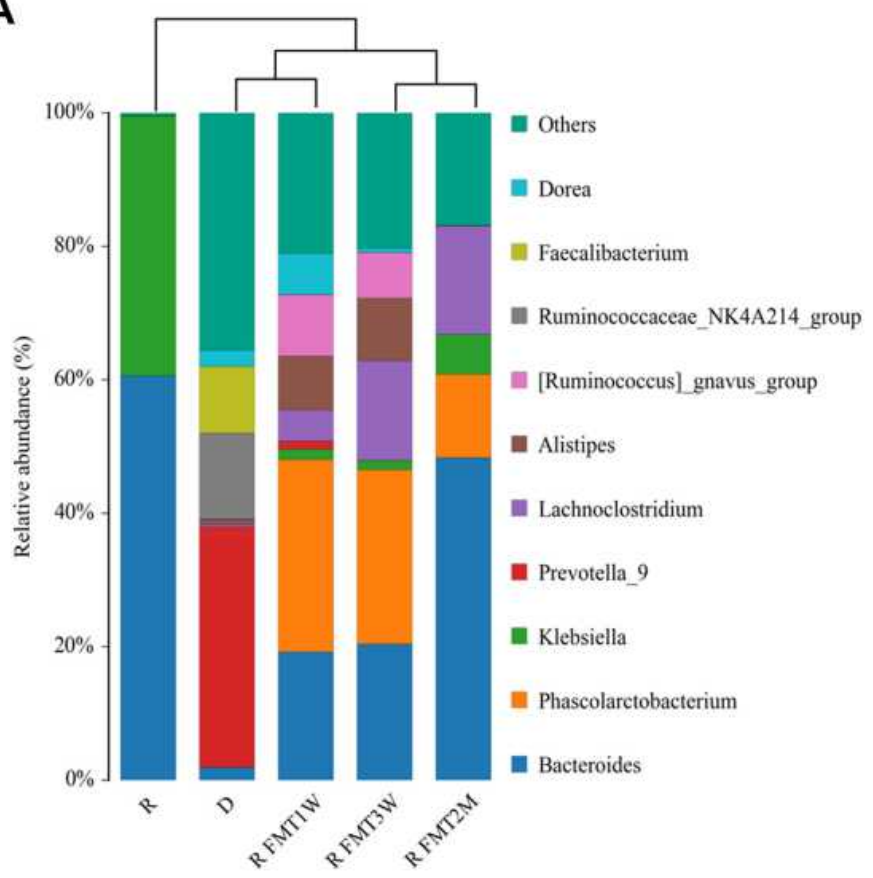

B
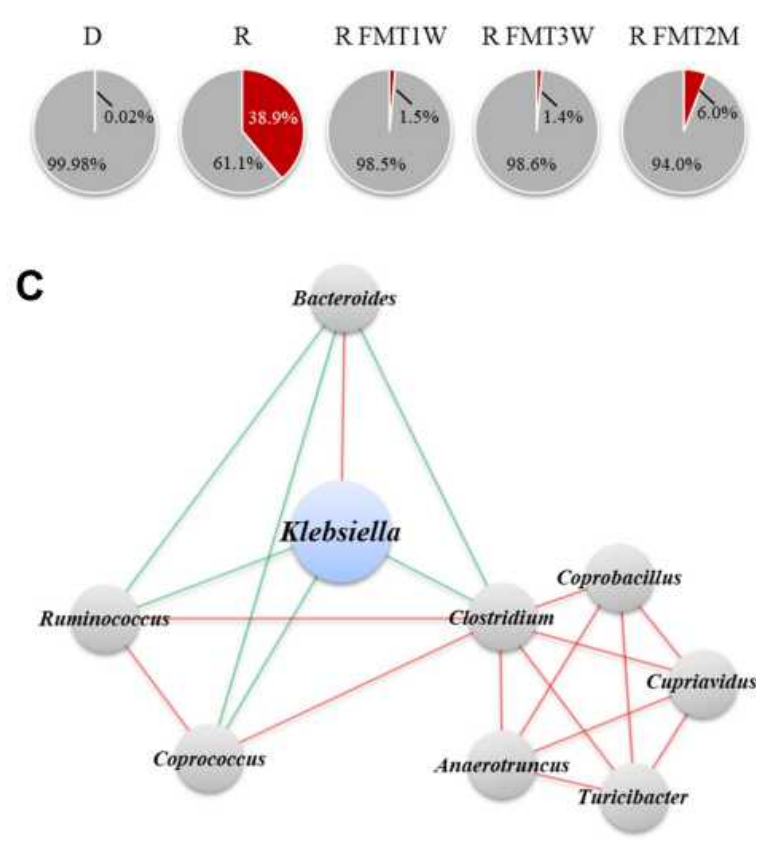

Figure 4 Gut microbial compositions of the donor and the patient pre- and post-FMT. (A) Cumulative histograms representing average distributions of major taxa at genus level. FMT IW, FMT 3W and FMT 2M represented I week, 3 weeks and 2 months after FMT. (B) Pie chart representing the relative abundance of Klebsiella (red). (C) Network diagrams showing the relationship between Klebsiella and other genera. Red indicated positive correlation and blue indicated negative correlation. Abbreviations: D, donor; $R$, recipient.

indicating that FMT may be an effective method for the treatment of ESBL+ Klebsiella pneumoniae infection. ${ }^{11}$ However, until now, the effect of FMT on CRKP infection has not been reported. To date, FMT has not yet been investigated with respect to its effect on the CRKP infection.

The CRKP we isolated from the patient was resistant against most antibiotics except tigecycline, fosfomycin and polymyxin. The patient probably developed resistance to carbapenem after the antibiotic therapy, since carbapenem antibiotics are routinely used for the renal transplant patient to prevent infection, and could provide selective pressure for the emergence of the resistance. According to data from European Centre for Disease Prevention and Control, more than a third of the Klebsiella pneumoniae isolates were resistant to at least one antimicrobial agent. ${ }^{12}$ Few available treatment options are left for patients infected with multidrug-resistant CRKP, and combination therapy or colistin is the common treatment methods. ${ }^{12}$ Although CRKP isolates were susceptible to polymyxin, considering its nephrotoxicity, ${ }^{13}$ it was not used for the treatment of the CRKP infection in order not to affect the recovery of renal function of the patient. Therefore, to our knowledge, the combination treatment of tigecycline, fosfomycin and meropenem that we used to treat the CRKP infection was probably the most suitable regimen. The unsatisfactory therapeutic effect of the combined therapy forced us to search for an alternative strategy such as FMT. One week after FMT, the patient's urine and anal swab cultures returned negative for CRKP, and 17 days after FMT, the incision showed complete healing. Moreover, the patient had no symptoms of infection two months after FMT.

Our results obtained from microbiome analysis showed that before FMT, Bacteroides and Klebsiella were predominant in the patient's fecal sample, and the relative abundance of Klebsiella was even up to $38.9 \%$. Klebsiella pneumoniae are ubiquitously distributed and considered as opportunistic pathogens inhabiting mucosal surfaces of intestines in healthy adults without inducing pathology. ${ }^{12}$ However, on some occasions, Klebsiella could disseminate from mucosae to other parts of the host, causing infections including pneumonia, urinary tract infections, bloodstream infections and sepsis. ${ }^{14}$ Clinical evidence and basic experiment suggest that liver abscess could also be induced when Klebsiella translocate across the intestinal epithelium. $^{15,16}$ To prevent rejection, the patient was placed on tacrolimus, mycophenolate mofetil, and 
prednisone after kidney transplantation. Among these drugs, tacrolimus is shown to have the effect of increasing intestinal permeability. ${ }^{17}$ Under this condition, it may be easier for CRKP to translocate across the intestinal epithelium to other places and induce infection. In addition, the CRKP strains isolated from the incision, urine, and fecal samples of the patient were shown to be exclusively grouped into ST11, indicating that the Klebsiella in the gut could be the most likely source of the CRKP detected in the other parts of the patient.

Obvious shift in microbial composition could be observed before and after FMT. The microbial composition of the patient post FMT resembled the donor composition. It should be noted that the relative abundance of genera such as Phascolarctobacterium and Lachnoclostridium increased after FMT, while the relative abundance of Klebsiella significantly decreased. Presumably, the removal of gut Klebsiella by FMT could be an important contributor to reduce the number of the CRKP in the patient's incision and urine. Similar to previous studies, we also found that the gut microbiota richness and diversity of the patient increased rapidly following FMT. ${ }^{18}$ The increased diversity of gut microbiota may have a positive effect on maintaining the stability of the colonic epithelial barrier and preventing Klebsiella pneumoniae from translocating, since Klebsiella pneumoniae was observed to barrier function and increase colonic permeability of luminal toxins, whereas other species such as Lactobacillus brevis could improve the tightness of the barrier. ${ }^{19}$ Moreover, the relative abundances of Ruminococcus, Coprococcus, and Clostridium were negatively correlated with that of Klebsiella, indicating that these genera may have distinct function from Klebsiella, and could partially limit the growth of Klebsiella. The increased abundance of Phascolarctobacterium and Lachnoclostridium after FMT may help maintain normal gut function. It has been reported that Lachnoclostridium could produce secondary bile acids through bile acid dihydroxylation activity, ${ }^{20}$ while Phascolarctobacterium could utilize succinate which induces diarrhea if accumulated in the intestine. ${ }^{21,22}$

Our research has several limitations. First, since this study is a case report, more clinical studies and basic experiments are needed to confirm the therapeutic effect of FMT on CRKP infection and explore its mechanisms. Secondly, though no recurrent infection was observed in the patient two months after FMT, the follow-up time was not long enough to investigate the sustained effect of FMT on CRKP infection. However, despite these limitations, this study demonstrated the therapeutic effect of FMT on CRKP infection, and may shine some light on the treatment of infections caused by multidrug-resistant CRKP for the patients after transplantation.

This study demonstrated the therapeutic effect of FMT on infections caused by CRKP in a renal transplant patient. Further studies are required to confirm the findings of this study.

\section{Abbreviations}

BUN, blood urea nitrogen; CRKP, carbapenem-resistant Klebsiella pneumoniae; CDI, Clostridioides difficile infection; FMT, fecal microbiota transplantation; IBS, irritable bowel syndrome; OTU, operational taxonomic unit; UC, ulcerative colitis.

\section{Code Availability}

Raw data were processed using the Quantitative Insight into Microbial Ecology (QIIME), version 1.8.0.

\section{Data Sharing Statement}

All data for this study are included in this published article.

\section{Ethics Approval and Informed Consent}

This study was conducted in accordance with the ethical guidelines based on the regulations of Henan Provincial People's Hospital affiliated to Zhengzhou University federal regulations. Written informed consent was obtained from the study participants for publication of this case report and any accompanying images. The transplanted kidney was from a donor following cardiac death, and it was donated voluntarily with written informed consent. This study was conducted in accordance with the Declaration of Istanbul.

\section{Author Contributions}

All authors made a significant contribution to the conception, study design, execution, acquisition of data, analysis and interpretation; took part in drafting, revising or critically reviewing the article; gave final approval for the version to be published; have agreed on the current journal; and agree to be accountable for all aspects of the work. 


\section{Funding}

This research was supported by the Henan Provincial Scientific and Technological Research Project (Grant No. 192102310036). Xin Li (funder) participated in research design, the writing of the paper, and data analysis.

\section{Disclosure}

The authors declare no conflict of interest.

\section{References}

1. Tzouvelekis LS, Markogiannakis A, Psichogiou M, Tassios PT, Daikos GL. Carbapenemases in Klebsiella pneumoniae and other Enterobacteriaceae: an evolving crisis of global dimensions. Clin Microbiol Rev. 2012;25(4):682-707.

2. van Duin D, Kaye KS, Neuner EA, Bonomo RA. Carbapenemresistant Enterobacteriaceae: a review of treatment and outcomes. Diagn Microbiol Infect Dis. 2013;75(2):115-120. doi:10.1016/j. diagmicrobio.2012.11.009

3. Diancourt L, Passet V, Verhoef J, Grimont PAD, Brisse S. Multilocus sequence typing of Klebsiella pneumoniae nosocomial isolates. J Clin Microbiol. 2005;43(8):4178-4182. doi:10.1128/JCM.43.8.41784182.2005

4. Shon AS, Bajwa RP, Russo TA. Hypervirulent (hypermucoviscous) Klebsiella pneumoniae: a new and dangerous breed. Virulence. 2013;4 (2):107-118. doi:10.4161/viru.22718

5. Fecal Microbiota Transplantation-standardization Study Group. Nanjing consensus on methodology of washed microbiota transplantation. Chin Med J. 2020.

6. Dai M, Liu Y, Chen W, et al. Rescue fecal microbiota transplantation for antibiotic-associated diarrhea in critically ill patients. Crit Care 2019;23(1):324. doi:10.1186/s13054-019-2604-5

7. Zhang T, Lu G, Zhao Z, et al. Washed microbiota transplantation vs. manual fecal microbiota transplantation: clinical findings, animal studies and in vitro screening. Protein Cell. 2020;11:251-266. doi:10.1007/s13238-019-00684-8

8. Johnsen PH, Hilpüsch F, Cavanagh JP, et al. Faecal microbiota transplantation versus placebo for moderate-to-severe irritable bowel syndrome: a double-blind, randomised, placebo-controlled, parallel-group, single-centre trial. Lancet Gastroenterol Hepatol. 2018;3(1):17-24. doi:10.1016/S2468-1253(17)30338-2

9. Aroniadis OC, Brandt LJ, Oneto C, et al. Faecal microbiota transplantation for diarrhoea-predominant irritable bowel syndrome: a double-blind, randomised, placebo-controlled trial. Lancet Gastroenterol Hepatol. 2019;4(9):675-685. doi:10.1016/S24681253(19)30198-0
10. Orenstein R, Dubberke E, Hardi R, et al.; PUNCH CD Investigators. Safety and durability of RBX2660 (Microbiota suspension) for recurrent clostridium difficile infection: results of the PUNCH CD Study. Clin Infect Dis. 2016;62(5):596-602. doi:10.1093/cid/civ938

11. Grosen AK, Povlsen JV, Lemming LE, Jørgensen SMD, Dahlerup JF, Hvas CL. Klebsiella pneumoniae faecal microbiota transplantation eradicated extended-spectrum beta-lactamase-producing from a renal transplant recipient with recurrent urinary tract infections. Case Rep Nephrol Dialysis. 2019;9(2):102-107. doi:10.1159/000502336

12. Bengoechea JA, Sa Pessoa J. Klebsiella pneumoniae infection biology: living to counteract host defences. FEMS Microbiol Rev. 2019;43(2):123-144. doi:10.1093/femsre/fuy043

13. Pogue JM, Ortwine JK, Kaye KS. Are there any ways around the exposure-limiting nephrotoxicity of the polymyxins? Int J Antimicrob Agents. 2016;48:622-626. doi:10.1016/j.ijantimicag.2016.11.001

14. Paczosa MK, Mecsas J. Klebsiella pneumoniae: going on the offense with a strong defense. Microbiol Mol Biol. 2016;80(3):629-661.

15. Siu LK, Yeh KM, Lin JC, Fung CP, Chang FY. Klebsiella pneumoniae liver abscess: a new invasive syndrome. Lancet Infect Dis. 2012;12(11):881-887. doi:10.1016/S1473-3099(12)70205-0

16. Tu YC, Lu MC, Chiang MK, et al. Genetic requirements for Klebsiella pneumoniae-induced liver abscess in an oral infection model. Infect Immun. 2009;77(7):2657-2671. doi:10.1128/ IAI.01523-08

17. Gabe SM, Bjarnason I, Tolou-Ghamari Z, et al. The effect of tacrolimus (FK506) on intestinal barrier function and cellular energy production in humans. Gastroenterology. 1998;115(1):67-74. doi:10.1016/S0016-5085(98)70366-X

18. Khanna S, Vazquez-Baeza Y, Gonzalez A, et al. Changes in microbial ecology after fecal microbiota transplantation for recurrent C. difficile infection affected by underlying inflammatory bowel disease. Microbiome. 2017;5(1):55. doi:10.1186/s40168-017-0269-3

19. Garcia-Lafuente A, Antolin M, Guarner F, Crespo E, Malagelada JR. Modulation of colonic barrier function by the composition of the commensal flora in the rat. Gut. 2001;48(4):503-507. doi:10.1136/ gut.48.4.503

20. Ridlon JM, Kang DJ, Hylemon PB, Bajaj JS. Gut microbiota, cirrhosis, and alcohol regulate bile acid metabolism in the gut. Dig Dis. 2015;33:338-345. doi:10.1159/000371678

21. Ferreyra JA, Wu KJ, Hryckowian AJ, Bouley DM, Weimer BC, Sonnenburg JL. Gut microbiota-produced succinate promotes C. difficile infection after antibiotic treatment or motility disturbance. Cell Host Microbe. 2014;16(6):770-777. doi:10.1016/j. chom.2014.11.003

22. Fernández-Veledo S, Vendrell J. Gut microbiota-derived succi-nate: friend or for in human metabolic diseases? Rev Endocr Metab Disord. 2019;20(4):439-447. doi:10.1007/s11154-019-09513-z
Infection and Drug Resistance

\section{Publish your work in this journal}

Infection and Drug Resistance is an international, peer-reviewed openaccess journal that focuses on the optimal treatment of infection (bacterial, fungal and viral) and the development and institution of preventive strategies to minimize the development and spread of resistance. The journal is specifically concerned with the epidemiology of antibiotic resistance and the mechanisms of resistance development and diffusion in both hospitals and the community. The manuscript management system is completely online and includes a very quick and fair peerreview system, which is all easy to use. Visit http://www.dovepress.com/ testimonials.php to read real quotes from published authors. 